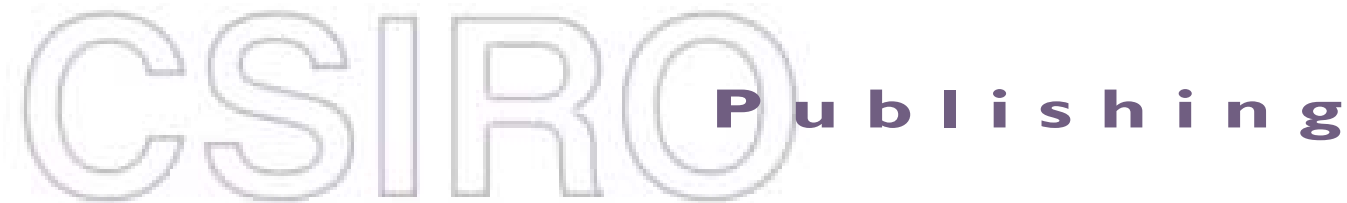

\section{Publications of the Astronomical Society of Australia}

Volume 19, 2002

(C) Astronomical Society of Australia 2002

An international journal of astronomy and astrophysics

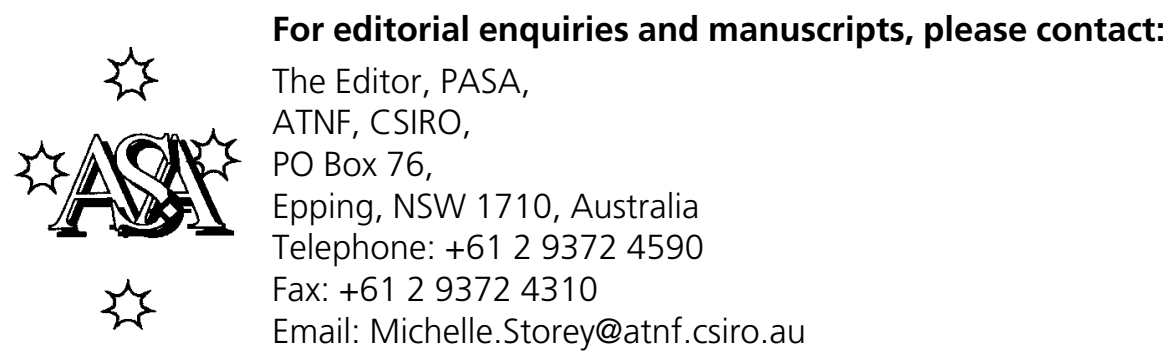

For general enquiries and subscriptions, please contact: CSIRO Publishing PO Box 1139 (150 Oxford St)

Collingwood, Vic. 3066, Australia

Telephone: +6139662 7666

Fax: +61 396627555

Email: publishing.pasa@csiro.au

C S I RO

PUBLISHING Published by CSIRO Publishing

for the Astronomical Society of Australia

www.publish.csiro.au/journals/pasa 


\title{
An Optical Design for an Antarctic 2 m Telescope with a Near IR Imager
}

\author{
P. R. Gillingham \\ Anglo-Australian Observatory, PO Box 296, Epping, NSW 1710, Australia \\ pg@aaoepp.aoo.gov.au \\ Received 2001 August 1, accepted 2001 November 27
}

\begin{abstract}
A study has been made of the optical performance to be expected from an Antarctic Ritchey Chrétien telescope with a $2 \mathrm{~m}$ diameter primary mirror imaging directly onto a detector array in the $\mathrm{K}$ and $\mathrm{L}$ infrared windows. Near diffraction limited performance is provided across a flat 30 arcmin diameter field by compensating the astigmatism and field curvature with a meniscus lens which also serves as the Dewar window. With baffling inside and Narcissus mirrors outside the Dewar, extraneous radiation can be kept to a low level.
\end{abstract}

Keywords: telescopes — infrared: general — instrumentation: photometers — methods: observational

\section{Introduction}

An extremely high priority for an Antarctic telescope with an aperture of around $2 \mathrm{~m}$ is wide field near infrared imaging since this is the application in which it can excel, compared with much larger telescopes at temperate sites. With the prospect of superb seeing at higher sites than the South Pole, the optics should not significantly degrade diffraction limited images. If sufficiently good rejection of extraneous radiation can be provided without re-imaging the telescope pupil onto a cold stop, then a simple, inexpensive, and very efficient system results.

In assessing the imaging performance of the system, two wavelength combinations were used: $2.3 \mu \mathrm{m}+$ $2.6 \mu \mathrm{m}$ and $3 \mu \mathrm{m}+4 \mu \mathrm{m}$. The former combination represents a filter covering that part of the $\mathrm{K}$ window which is most useful in Antarctica, being unaffected by $\mathrm{OH}$ sky lines (Ashley et al. 1996; Nguyen et al. 1996). The second represents the $\mathrm{L}$ window, one in which the low sky background in Antarctica will be very beneficial (Phillips et al. 1999).

\section{Optical System}

\subsection{Telescope Layout}

A primary mirror diameter of $2 \mathrm{~m}$ and focal length $3 \mathrm{~m}$ is combined with a secondary mirror giving a final focal length of $12 \mathrm{~m}$. The conic constants of both the primary and secondary are optimised to give best performance across a field 30 arcmin in diameter, resulting in a Ritchey Chrétien prescription. The mirror diameter was chosen as a compromise between performance and cost. Its focal ratio, f/1.5, was recommended by a telescope manufacturer as the optimum, taking into consideration the cost of figuring the primary, the size and cost of the enclosure, and the size of the secondary mirror (smaller for a faster primary, making fast tip-tilt control easier). The focal length was chosen to match a 30 arcmin field diameter to the diagonal of a $4 \mathrm{k} \times 4 \mathrm{k}$ detector array with
$0.018 \mathrm{~mm}$ square pixels. Then each pixel is equivalent to about 0.31 arcsec, which is a reasonable match to the Airy disc diameters, from $0.54 \operatorname{arcsec}$ at $2.3 \mu \mathrm{m}$ to 0.94 arcsec at $4 \mu \mathrm{m}$. Figure 1 shows the layout of the optics with no instrument.

\subsection{Image Quality of Basic Telescope}

The Ritchey Chrétien system has no coma but suffers from astigmatism and field curvature. At 15 arcmin field radius, on the best matching spherical focal surface, ray tracing gives an image spread in excess of 1 arcsec diameter and a near infrared image is very significantly degraded compared with the diffraction limited case for the $2 \mathrm{~m}$ aperture. The inset in Figure 1 shows the point spread function (PSF) at this field radius derived from ray tracing with allowance for diffraction, for combined wavelengths 2.3 and $2.6 \mu \mathrm{m}$. The Strehl ratio (SR, defined as the ratio of the peak intensity to that for the perfect diffraction limited case) is 0.18 . The cross structure of the PSF is characteristic of astigmatism midway between the sagittal and tangential foci.

\subsection{Image Quality with Aberration Correction by Dewar Window}

A lens or aspheric plate set ahead of the telescope focus can considerably reduce the aberrations of the Ritchey Chrétien telescope (Gascoigne 1965). This element can also serve as the vacuum sealing window to the cryogenic enclosure needed for the detector. Figure 2 shows the optical layout with a meniscus lens (of calcium fluoride) optimised for these dual functions. Note that the curvature of the meniscus is favourable to supporting atmospheric pressure with the window, while placing the calcium fluoride only in compression. With both surfaces of the window spherical, the images on a flat focal surface are so much improved that the SR for an image 15 arcmin off axis with combined wavelengths 2.3 and $2.6 \mu \mathrm{m}$ is now 0.51 . The PSF is shown inset at the top of Figure 2 


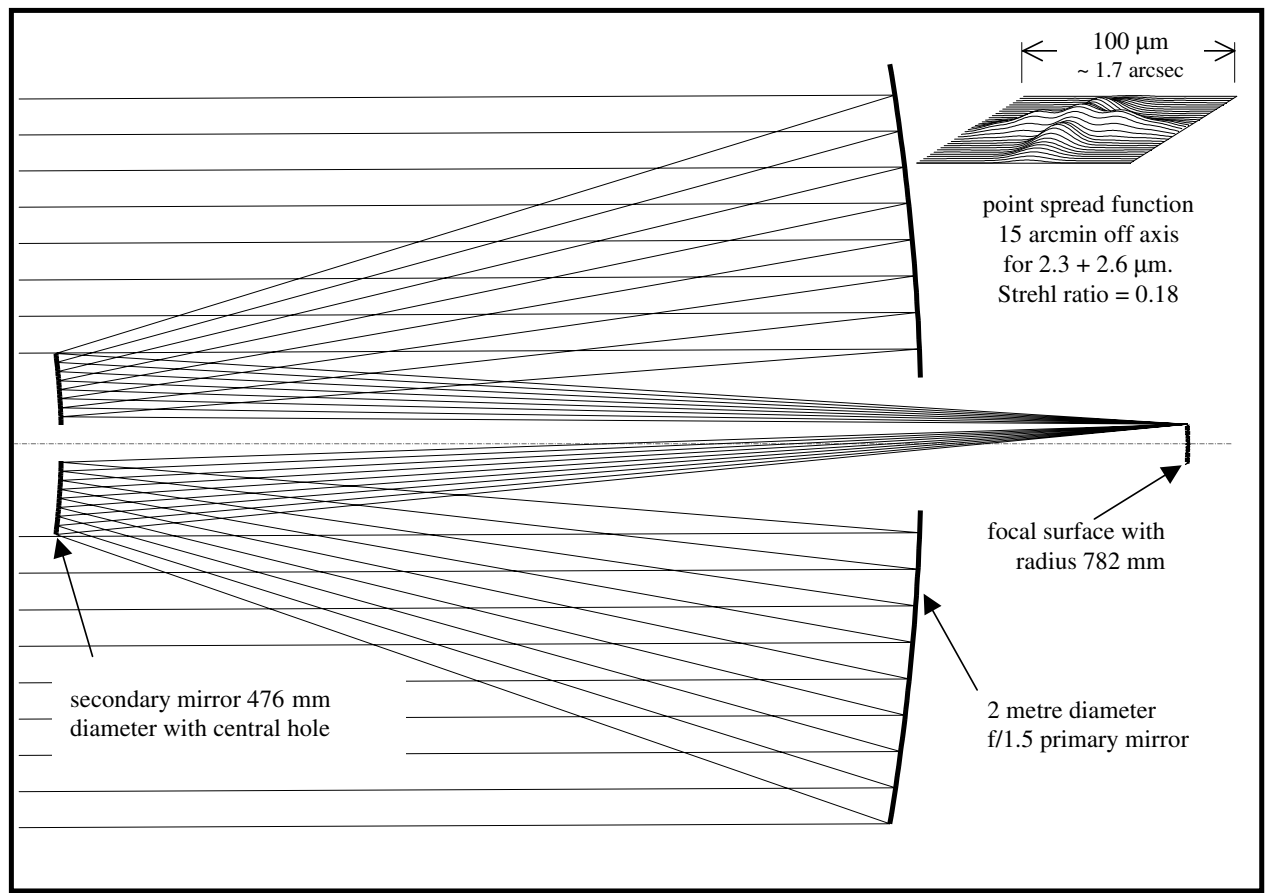

Figure 1 Optical layout of bare telescope showing point spread function at edge of 30 arcmin diameter field.

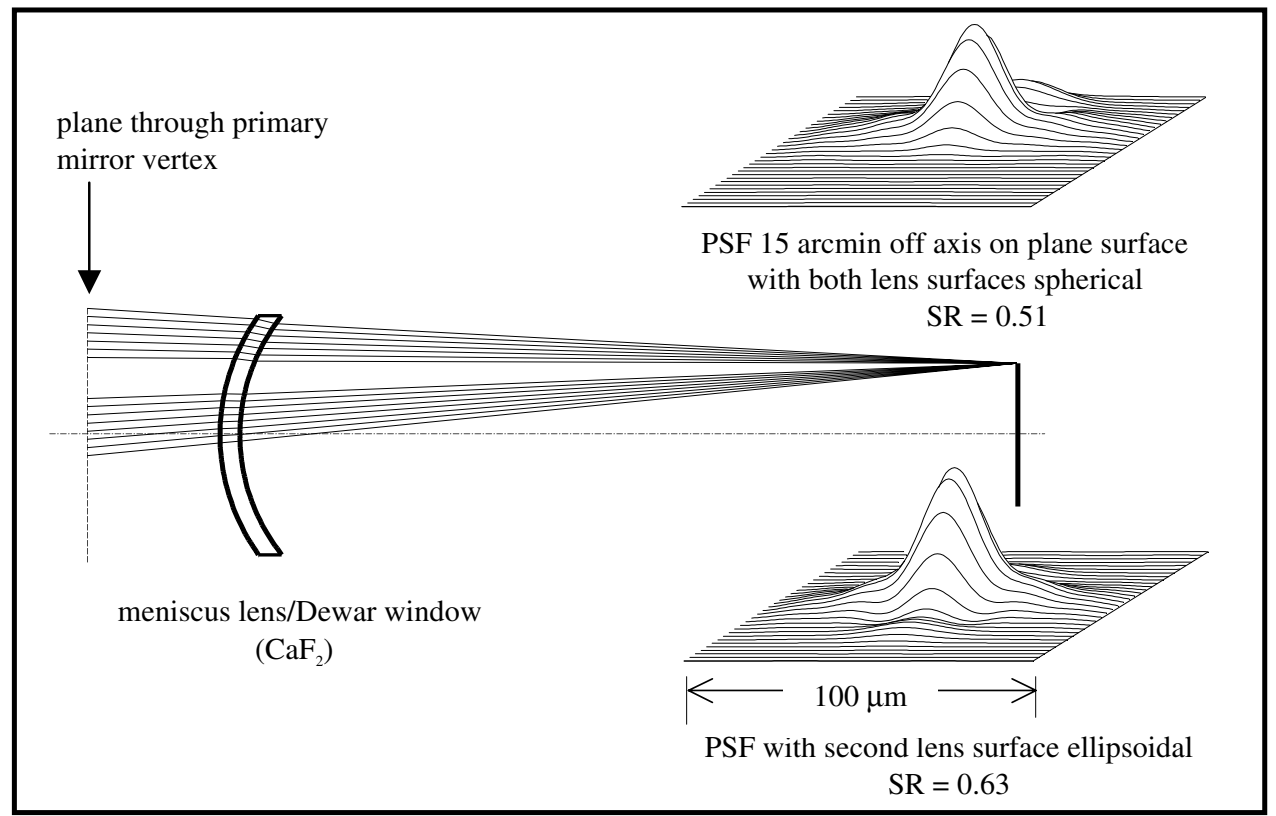

Figure 2 Layout for meniscus lens and detector with images produced at edge of 30 arcmin diameter flat field.

and the optical design details are given in Table 1. Further improvement can be made by figuring the concave surface of the meniscus lens to a conic (an ellipsoid). The SR at the edge of the field is then 0.63 (see PSF inset at the bottom of Figure 2).

Better performance still is possible across a field with 30 arcmin diagonal if the focal surface is tiled with 4 detector arrays and each is tilted about its diagonal to better conform to a curved focal surface. With the meniscus lens optimised to suit this arrangement, illustrated in
Figure 3, the worst SR within the 21 arcmin square field for combined wavelengths 2.3 and $2.6 \mu \mathrm{m}$ is 0.89 .

For longer wavelengths where diffraction is more dominant, the SR is, of course, higher in each case.

\section{Minimising Extraneous Radiation}

\subsection{Radiation from Narcissus Mirrors}

With no provision for imaging the telescope pupil onto a cold stop, extraneous radiation will reach the detector 
Table 1. Optical prescription for system with meniscus having both surfaces spherical

\begin{tabular}{llrlcc}
\hline Surface & $\begin{array}{c}\text { Radius of } \\
\text { curvature }(\mathrm{mm})\end{array}$ & $\begin{array}{c}\text { Separation } \\
(\mathrm{mm})\end{array}$ & Material & $\begin{array}{c}\text { Diameter } \\
(\mathrm{mm})\end{array}$ & Conic constant \\
\hline Primary mirror & -6000.00 & -2259.77 & Mirror & 2000.0 & -1.043 \\
Secondary mirror & -1973.34 & 2259.77 & Mirror & 476.0 & -3.193 \\
At primary vertex & Infinity & 100.00 & & 192.3 & 0 \\
Meniscus 1st surface & 182.76 & 15.00 & $\mathrm{CaF}_{2}$ & 180.0 & 0 \\
Meniscus 2nd surface & 173.78 & 585.00 & & 180.0 & 0 \\
Image plane & Infinity & & & & 0 \\
\hline
\end{tabular}

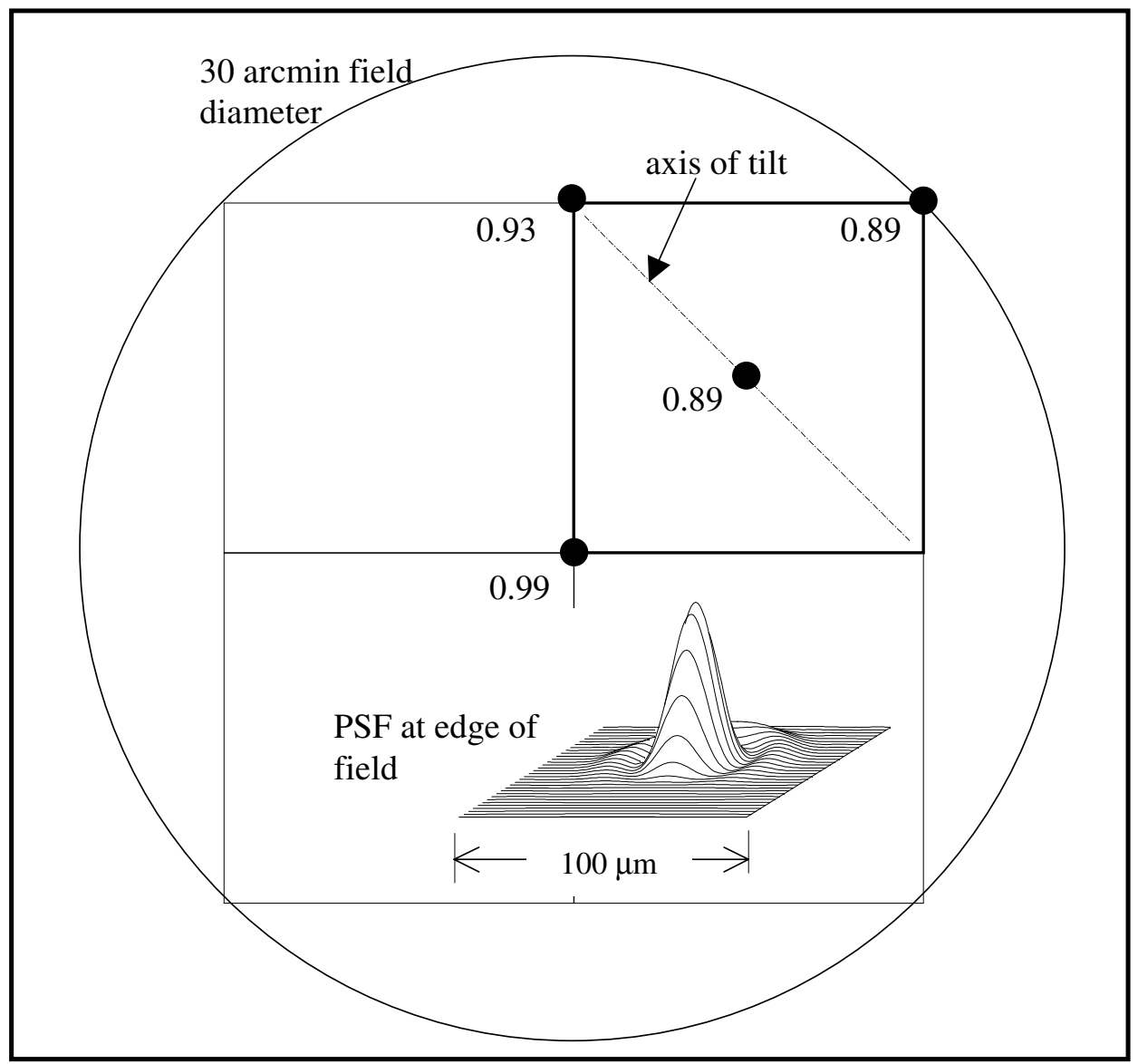

Figure 3 Mosaic of four arrays each $2 \mathrm{k} \times 2 \mathrm{k}$ with pixels 0.018 square. Each array is tilted by $1^{\circ}$ around an axis inclined as indicated for the top right array, to give a best fit to the curved focal surface. For each of the 4 positions in the field, Strehl ratios are shown for $2.3+2.6 \mu \mathrm{m}$.

from a bigger solid angle than is the case in most astronomical infrared cameras. However, the very low ambient temperature near the ground in Antarctica compared with temperatures higher in the atmosphere eases this problem. In particular, Narcissus mirrors - concave mirrors outside the Dewar, in which the detector 'sees' radiation predominantly from within the Dewar - can be much more effective than at temperate sites.

At the South Pole in winter, measurements of the near infrared sky spectrum (Phillips et al. 1999; Hidas et al. 2000) show that the spectral intensities (in units of $\mathrm{Jy} \operatorname{arcsec}^{-2}$ ) at $2.4 \mu \mathrm{m}$ and $3.8 \mu \mathrm{m}$ (where sky emissivity is near minimum in the $\mathrm{K}$ and $\mathrm{L}$ windows) were, respectively, about 10 times and 0.7 times the spectral intensities of a black body at the ground level temperature $\left(-70^{\circ} \mathrm{C}\right)$. With a Narcissus mirror coated in silver or gold, emissivity through this wavelength range is about $2 \%$. Conservatively, another $4 \%$ should be added for the reflectivity of the two (anti-reflection coated) surfaces of the Dewar window which will be seen by the detector reflected in the Narcissus mirror. Allowing 6\% as the apparent emissivity of a Narcissus mirror surface, the spectral intensities from the mirror will be about $0.6 \%$ and $9 \%$ of those from the sky at $2.4 \mu \mathrm{m}$ and $3.8 \mu \mathrm{m}$ respectively. Integrated over the $\mathrm{K}$ or $\mathrm{L}$ windows, the intensities from the mirror will be somewhat smaller as a proportion of the sky intensity. 


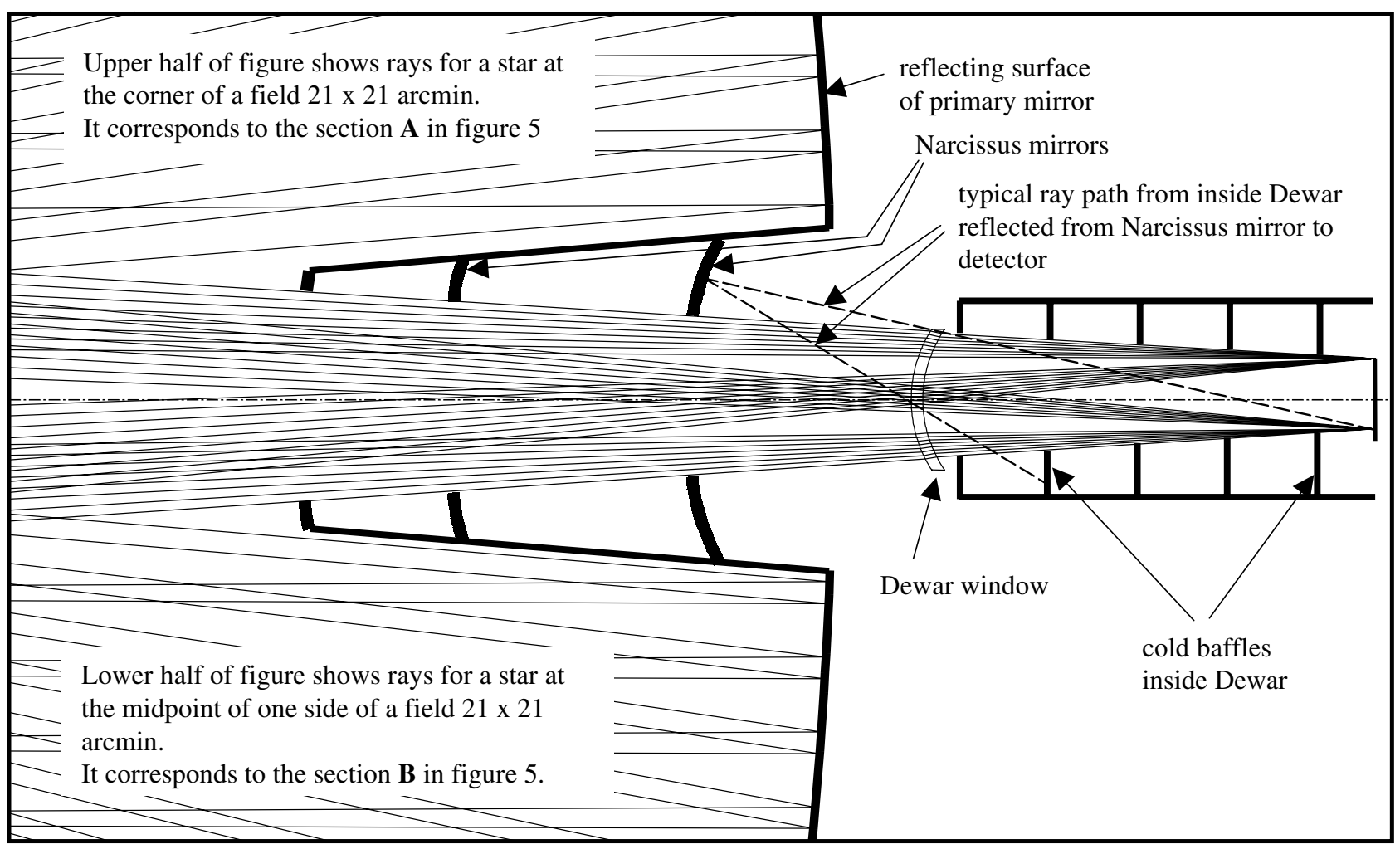

Figure 4 Layout of cold baffles in Dewar and Narcissus mirrors in central sky baffle to minimise extraneous radiation reaching detector.

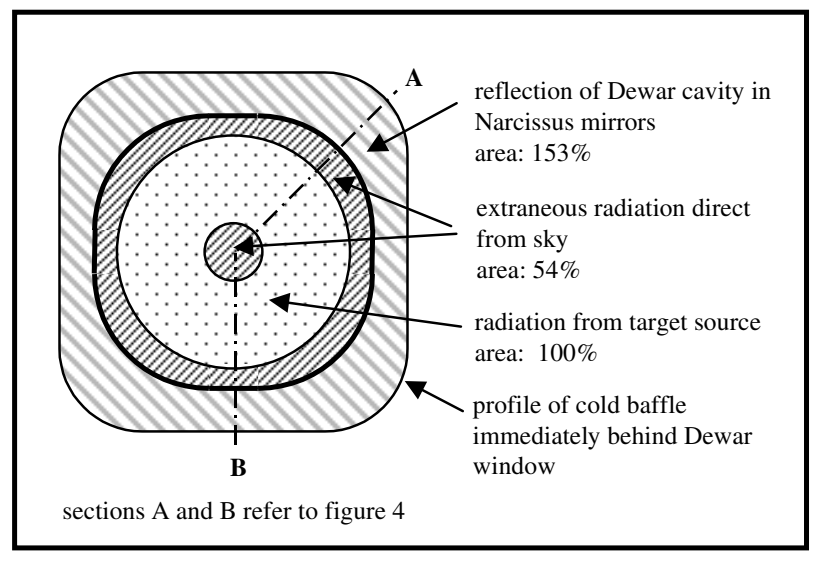

Figure 5 Cross section of beam approaching centre of detector, showing proportions of solid angles of extraneous radiation in comparison with radiation from target, with baffles and Narcissus mirrors as in figure 4.

In winter, at higher altitude sites than the South Pole, both the ground level temperature and the effective atmosphere temperature will typically be lower, but the ratios derived above should not change enough to affect the conclusions.

\subsection{Effect of Baffles and Narcissus Mirrors}

Figure 4 shows how baffles inside the Dewar and Narcissus mirrors outside the Dewar can be positioned to minimise extraneous radiation, without vignetting a square field with 30 arcmin diagonal. Figure 5 is a cross section through the beam approaching the centre of the detector, identifying the source of the radiation out to where baffles inside the Dewar reduce the radiation to an insignificant level.

The area of each zone, as a percentage of the useful area, i.e. the area of the annulus representing radiation from the target focused onto the detector, is indicated on the figure. For points away from the detector centre, the various profiles in Figure 5 would be translated with respect to each other but the areas of the zones would be unchanged. Radiation is reflected from the Narcissus mirrors over an area 1.53 times larger than the useful area. Allowing for the apparent intensities of reflections from the Narcissus mirrors, the increases over the unavoidable contributions by sky radiation at $2.4 \mu \mathrm{m}$ and $3.8 \mu \mathrm{m}$ are the equivalent of about $0.9 \%$ and $14 \%$ respectively.

The area representing extraneous radiation direct from the sky, including that from the hole through the secondary, is 0.54 times the useful area. Adding the contributions from Narcissus mirrors and from the sky, the extraneous radiation increases background by about $55 \%$ and $68 \%$ at the two wavelengths considered.

For a smaller angular field, the internal profiles of the baffles within the Dewar and of the Narcissus mirrors can be shrunk, reducing the extraneous radiation roughly in proportion to the linear size of the field. Thus with a 15 arcmin diagonal, corresponding to a single $2 \mathrm{k} \times 2 \mathrm{k}$ array with $0.018 \mathrm{~mm}$ pixels, extraneous radiation would increase background by about $28 \%$ and $34 \%$ at the two wavelengths. 


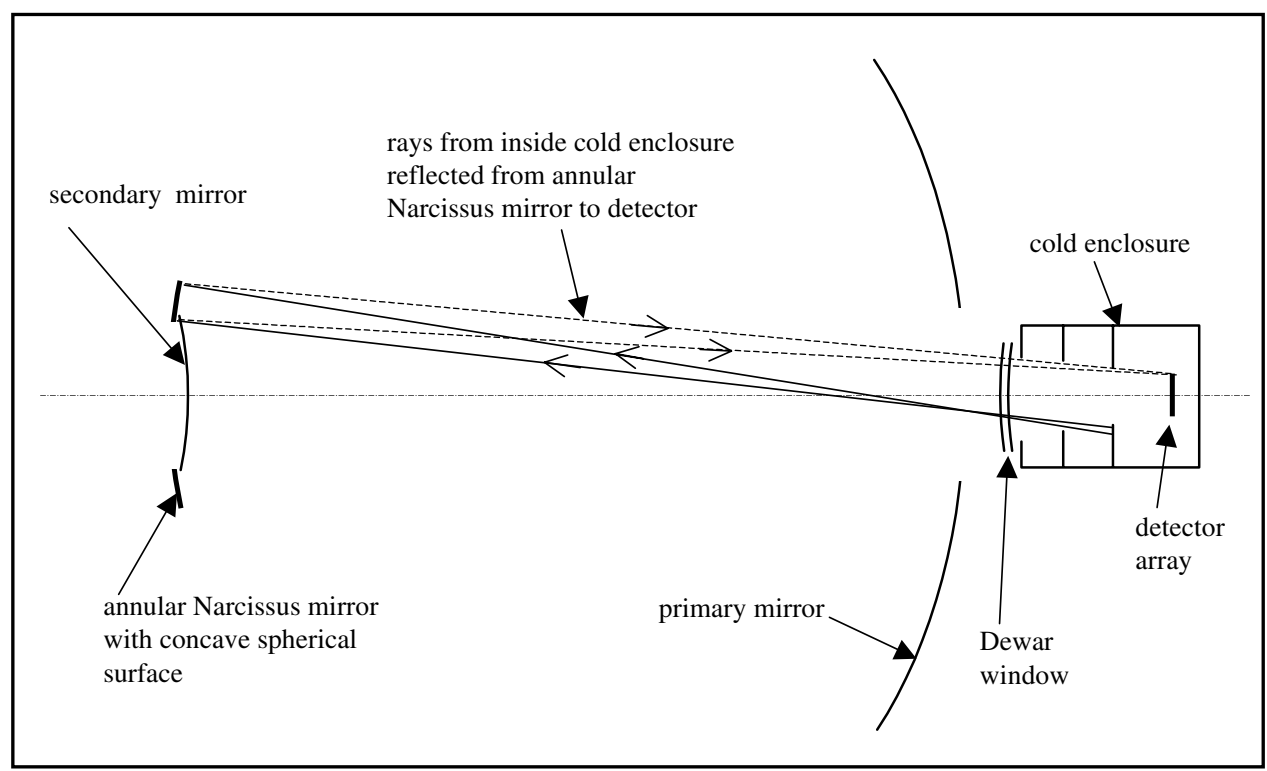

Figure 6 Operation of Narcissus mirror surrounding secondary mirror - not to scale.

The extraneous radiation direct from the sky could be eliminated altogether with the addition of an annular Narcissus mirror surrounding the secondary mirror and another in place of the hole in the secondary. The annular mirror would obstruct less than $1 \%$ of the useful radiation on its way to the primary mirror. Then the increases in background for the full 21 arcmin square field would be only about $2 \%$ and $20 \%$ at the two wavelengths. Figure 6 is a sketch indicating how the Narcissus mirror surrounding the secondary would operate. The accuracy with which these mirrors would have to be aligned would be higher than for the Narcissus mirrors within the central baffle but should be readily met, being still much less than the requirements for co-aligning the main telescope mirrors.

\section{Conclusion}

This design is as simple as it could be to provide wide field near infrared imaging without significantly compromising resolution or sensitivity. With so few optical surfaces, it will have very high efficiency and freedom from stray light arising from internal reflections. The only additional optical provision would be for filters ahead of the detector.

An initial version with a single detector array $1 \mathrm{k} \times 1 \mathrm{k}$ or $2 \mathrm{k} \times 2 \mathrm{k}$ could be upgraded to accept a mosaic with up to about $4 \mathrm{k} \times 4 \mathrm{k}$ pixels or even more to exploit the whole of the circular field 30 arcmin in diameter.

\section{Acknowledgments}

Peter McGregor suggested, at a workshop held on 4 May 2001 at the University of New South Wales to discuss science with an Antarctic infrared telescope, that a near infrared camera without a cold stop and employing a Narcissus mirror might offer adequate rejection of extraneous radiation. His suggestion prompted this study.

\section{References}

Ashley, M. C. B., et al. 1996, PASP, 721

Gascoigne, S. C. B. 1965 , Obs, 85,79

Hidas, M. G., Burton, M. G., Chamberlain, M. A., \& Storey, J. W. V. 2000, PASA, 17, 260

Nguyen, H. T., et al. 1996, PASP, 108, 718

Phillips, A., et al. 1999, ApJ, 527, 1009 